\title{
PRESCRIPCIÓN DE LA RECREACIÓN, UN ELEMENTO CLAVE ENTRE LA SALUD Y ENFERMEDAD
}

\author{
PRESCRIPTION OF RECREATION, A KEY ELEMENT BETWEEN HEALTH AND DISEASE
}

\author{
Henry Humberto León A. MD. ${ }^{1}$ \\ Nathaly Andrea Ospino D. ${ }^{2}$
}

\section{Resumen}

\begin{abstract}
Las enfermedades crónicas no transmisibles son la primera causa de muerte en Colombia y el mundo, por lo cual, cualquier esfuerzo por detener su continuo avance es útil y pertinente; la recreación como proceso facilitador contribuye activamente con este compromiso, desde la implementación programas recreativos como complemento de la educación, hasta los beneficios que junto al ejercicio físico (recreación terapéutica) tiene en el tratamiento de enfermedades como la obesidad, la diabetes, la dislipidemia, la hipertensión y la enfermedad cardiovascular, esto nos ha permitido proponer el concepto de "prescripción de la recreación" como el elemento integrador de la recreación y la salud, en una época en la que necesitamos más que nunca de herramientas que combatan las enfermedades crónicas no transmisibles en el mundo.
\end{abstract}

Palabras clave: salud, enfermedad, recreación, prescripción de la recreación.

Abstract

Non-communicable chronic diseases are the most important cause of death in Colombia and worldwide, so any effort to stop its further progression is useful and appropriate, the recreation as facilitator processes actively contributes to this commitment, since the implementation of recreational programs in addition to education, to the benefits along with exercise (therapeutic recreation) have in the treatment of diseases like obesity, diabetes, dyslipidemia, hypertension and cardiovascular disease, this has allowed us to propose the concept of "recreation's prescription" as the integrating element of recreation and health, at a time when we need more tools than ever to combat noncommunicable diseases in the world.

Key words: health, disease, recreation, prescription of recreation.

Fecha de recepción: 1 de Octubre de 2010

Fecha de aprobación: 29 de Octubre de 2010

\footnotetext{
${ }^{1}$ Licenciado en Educación Física de la Universidad Pedagógica Nacional, Médico Cirujano de la Universidad Nacional de Colombia. Docente tiempo completo de la Universidad Santo Tomás y docente catedrático de las licenciaturas en Educación Física y Licenciatura en Recreación de la Universidad Pedagógica Nacional sede Bogotá. preparacionfisica2@gmail.com

${ }^{2}$ Estudiante de noveno semestre de la Licenciatura en Recreación de la Universidad Pedagógica Nacional sede Bogotá.nathalyospino2005@hotmail.com
} 


\section{Introducción}

En la actualidad la gran mayoría de enfermedades en Colombia y en el mundo tienen una fuerte asociación con el sedentarismo y la falta de hábitos de vida saludables (HH, Melo \& Zea, 2009), enfermedades como la obesidad, la diabetes, la hipertensión y la enfermedad cardiovascular se encuentran dentro de las patologías más prevalentes en nuestro medio y corresponden a las primeras causas de morbilidad y mortalidad (Lloyd-Jones, et al., 2009) (Ministerio de Protección Social, 2008). La gran mayoría de los que padecen este tipo de enfermedades conviven con ella por el resto de su vida, sin posibilidades de curación, y además por su carácter no transmisible (aunque socialmente comportamientos y hábitos fundamentales en el desarrollo de la enfermedad si tengan un carácter transmisible), son denominadas como "enfermedades crónicas no transmisibles" (ECNT), las cuales tienen una prevalencia en aumento.

Algunas de las causas que llevan al desarrollo de ECNT, están fuertemente asociadas a una falta de políticas nacionales que apunten a la prevención oportuna (Horton, 2005), y también a una falta de condiciones que apunten al desarrollo de adecuados hábitos de vida, optimización en los servicios de salud y un adecuado seguimiento de la prevalencia de enfermedades crónicas en nuestro país (Lucumí, et al., 2008). Epidemiológicamente lo que ha demostrado ser la intervención costo efectiva más importante en el manejo las ECNT, es la actividad física regular (Warburton, Nicol \& Bredin, 2006) y en la actualidad a la par con el desarrollo del ejercicio físico evoluciona también un nuevo concepto, el de la recreación terapéutica, como forma de ejercicio físico y salud.

En nuestra sociedad y a la par con la problemática desencadenada por las ECNT está el rápido desarrollo que han tenido las enfermedades mentales y de estas especialmente la depresión, la cual ha venido cobrando una gran importancia; tan grave es el problema que se estima que para el año 2020 esta podría ser la segunda causa de morbilidad en nuestro país (Gómez-Restrepo, et al., 2004), lo cual ha hecho que se incrementen sus esfuerzos por su control y prevención.

La presente revisión intenta por lo tanto relacionar los avances que en el entorno de la recreación se han hecho con el fin de apoyar los programas de salud pública, (especialmente a partir del concepto de recreación terapéutica) y ponerlos al alcance de estudiantes y profesionales en el área de la recreación y la educación física en general.

\section{Recreación y programas de educación}

Los programas de recreación son una parte fundamental en el complemento de la educación, razón por la cual es posible plantear que desde los primeros años de educación esta puede participar de forma activa en la prevención de la enfermedad.

La evidencia ha logrado demostrar que las comunidades con más actividades artísticas, entrenamiento físico y actividades de tipo recreativo llevan al desarrollo de niños más saludables, de esta manera un estudio realizado por el departamento de Salud de Ohio logró demostrar una asociación positiva, entre el dinero invertido en actividades de recreación y el estado de salud de la niños (Rogers \& Zaragoza-Lao, 2003). Otros modelos de programas organizados, no competitivos que llevan a la adecuada utilización del tiempo libre han permitido incrementar la actividad física en los niños, algunas de las intervenciones tienen que ver con la participación de los niños en granjas experimentales, programas para el adecuado uso del tiempo libre y de fundamentación deportiva (Kien \& Chiodo, 2003).

Más adelante durante la adolescencia el número de actividades deportivas y recreativas en la que participan los jóvenes decae con el tiempo, se ha establecido en promedio que la probabilidad de que se mantengan dichas actividades en un periodo de 4 años es realmente baja, (Aaron, Storti, Robertson, Kriska \& LaPorte, 2002), especialmente en el género femenino donde las mujeres prefieren otras actividades a la recreación.

Las actividades recreativas traen beneficios, ya que se asocian a estilos vida saludable, bienestar físico, mejora de la autoestima, mejora de las relaciones con los padres, al incremento de la función cognitiva y a la disminución del aburrimiento entre otros, esto hace que sea necesario pensar en cambios sociales que favorezcan los programas de recreación (Ott, et al., 2006). En los casos de la participación de niños en condición de discapacidad en actividades deportivas y recreativas se promueve la inclusión social, con el objetivo de minimizar el desacondicionamien- 
to y optimizar el funcionamiento físico y aumenta la sensación de bienestar (Murphy \& Carbone, 2008).

\section{Recreación, actividad física y salud}

La recreación como forma de actividad física ha demostrado efectos benéficos, especialmente en el tratamiento de enfermedades crónicas no transmisibles, cuando hablamos de recreación para la salud, hablamos de recreación terapéutica, dando un énfasis fuerte en la construcción de hábitos de vida saludable, pero sobre todo, para la construcción de dinámicas psicosociales que procuren el bienestar individual y social. Así, la intención primaria de los procesos de recreación en el marco de la salud, son los de coadyuvar en la dinámica de integración social para la disminución de factores que afectan la calidad de vida.

La enfermedad como elemento antagónico de la salud, surge cuando se altera la homeostasis (equilibrio) y muchos de los elementos que allí participan tienen que ver con el mal aprovechado uso del tiempo y el inadecuado manejo de los hábitos de vida saludables.

Obesidad: resultados del Instituto Colombiano de Bienestar Familiar indican que cerca de la mitad de la población adulta de Colombia presenta un peso elevado para la estatura, con una prevalencia de $32.3 \%$ de sobrepeso y $13.8 \%$ de obesidad (Instituto Nacional de Salud, 2005), más preocupante que esto, está el hecho de que la población de niños con sobrepeso se ha triplicado en las últimas tres décadas en el mundo (Hill \& Wyatt, 2005), lo cual sin duda es el resultado de hábitos sedentarios adquiridos en la infancia, probablemente asociados al uso excesivo de la televisión, el computador y los videojuegos como forma de recreación de pobre gasto calórico (Norman, Schmid, Sallis, Calfas \& Patrick, 2005).

Diabetes: en sólo los Estados Unidos, la diabetes Mellitus tipo 2 (DMT2) afecta cerca de 16 millones de personas (Bassuk \& Manson, 2005). A esta enfermedad se le atribuye un aumento del riesgo coronario (infarto agudo del miocardio) de tres a siete veces en comparación con una persona no diabética, sin contar otras múltiples consecuencias como la ceguera, la insuficiencia renal crónica, las alteraciones de los vasos sanguíneos y de los nervios. Múltiples estudios han demostrado que las personas físicamente activas tienen una reducción del riesgo cardiovascular asociado a DMT2 entre el 30 a 50\% (Bassuk \& Manson, 2005), con lo que programas de recreación activa podrían contribuir de una forma activa a la lucha contra la enfermedad.

Dislipidemia: el término dislipidemia se refiere a un aumento en la cantidad de colesterol de baja densidad (C-LDL), un aumento de los triglicéridos (TG), con una disminución del colesterol de alta densidad (C-HDL). Esta combinación se asocia fuertemente a la formación de ateromas (placas de grasa en el interior de las arterias), y estos a una mayor probabilidad de enfermedad cardiovascular. El ejercicio regular y una adecuada alimentación ha comprobado ampliamente que mejora los niveles de C-HDL, al tiempo que reduce los valores de C-LDL y TG, por lo tanto una reducción del riesgo cardiaco (Thompson, et al., 2003).

Hipertensión: la hipertensión o valores de presión arterial superiores a 140/90 milímetros de mercurio (mm/Hg), es una enfermedad silenciosa que lentamente lesiona vasos sanguíneos como los de la retina y el riñón, y que a la vez produce cambios anatómicos en el corazón que alteran su normal funcionamiento, su origen en gran medida es desconocido, pero en la actualidad se cree que el exceso de tejido adiposo, especialmente visceral juega un papel importante en el desarrollo de la enfermedad, y que por lo tanto la actividad física puede participar en la prevención y tratamiento de esta enfermedad debido entre otros a la disminución de tejido adiposo (Mathieu, Poirier, Pibarot, Lemieux \& Després, 2009).

Enfermedad cardiovascular: en conjunto la obesidad, la diabetes, la dislipidemia y la hipertensión son factores que junto a otros como el hábito de fumar y el sedentarismo predisponen a la aparición de enfermedades que afectan el sistema cardiovascular, especialmente al sistema vascular del corazón (las arterias coronarias).

En Colombia y en general a nivel mundial las enfermedades coronarias constituyen la primera causa de muerte por encima de los 45 años esto especialmente dado por la presencia de hábitos no saludables dentro de los que se destaca el sedentarismo, el tabaquismo y el consumo de alcohol, los cuales mantienen asociación con otras enfermedades; al 
tiempo que se ha comprobado que tanto hombres como mujeres que incrementaron sus niveles de actividad física han disminuido su riesgo de morir por enfermedad cardiovascular entre un 20\% a un 35\% (Warburton, et al., 2006).

Otras enfermedades: luego de bastante tiempo ha sido posible demostrar muchos otros beneficios de la actividad física; algunos tipos de cáncer como el de colon presentan una reducción del riesgo de padecerlo entre el 30 a 40\% y en el caso del cáncer de seno hasta un 30\% (Warburton, et al., 2006). En la osteoporosis se ha demostrado un significativa mejoría de la calidad ósea, asociada a una disminución del riesgo de caídas (Todd \& Robinson, 2003), dentro del manejo de muchas enfermedades mentales como la depresión o ansiedad, el ejercicio y las actividades recreativas se ha convertido en un pilar fundamental (Richardson, et al., 2005), en este sentido los programas de recreación social se enfocan en ayudar a la gente en el desarrollo de sus relaciones personales, varios estudios han demostrado el impacto de los programas de recreación en las relaciones sociales, adecuado uso del tiempo libre y la satisfacción en general, en personas afectadas por enfermedades mentales (Petryshen, Hawkins, \& Fronchak, 2001).

La recreación terapéutica, inicialmente por la sola gratificación y bienestar que genera, y posteriormente por los beneficios que asociados a la práctica de actividad física acarrea, debe ser considerada como una herramienta clave en el campo de la medicina preventiva y la salud, concepto que denominaremos "prescripción de la recreación".

\section{Recreación y espacio}

Una de las características de la cual requieren la proximidad y el acceso a la infraestructura necesaria para la práctica de la actividad física son determinantes clave en los programas de recreación (Dahmann, Wolch, Joassart-Marcelli, Reynolds, \& Jerrett, 2010). Sin embargo, parques, recreación y salud pública necesita más que sólo incrementar el número de parques o las personas que los visitan, debe haber un beneficio medible para la salud y la calidad de vida (Kruger, 2008).

\section{Conclusión}

Aunque poco tenida en cuenta, la recreación debería ser vista como un elemento clave en la medicina con fines preventivos, esto debido a los múltiples beneficios que trae su implementación en el mejoramiento de la salud y el control de la enfermedad; bajo esta premisa es necesario propender porque nuevos estudios sociales sean realizados, los cuales fortalezcan el conocimiento, la comprensión y la pertinencia de los programas, y den vida entonces a un nuevo concepto como el de la "prescripción de la recreación", en donde se reúnan no sólo los beneficios, sino también otras variables, como la forma, la cantidad o la intensidad de los estímulos recreativos con miras al mantenimiento de la salud y la prevención de la enfermedad. A conseguir cumplir este objetivo son llamados los Licenciados en Recreación a liderar el proceso investigativo y a proponer intervenciones útiles, concretas y aplicables en nuestra sociedad.

\section{Referencias}

Aaron D., Storti K., Robertson R., Kriska A., LaPorte R. (2002). Longitudinal study of the number and choice of leisure time physical activities from mid to late adolescence, Arch Pediatr Adolesc Med, 156, 1075-1080.

Bassuk S., Manson J. (2005). 'Epidemiological evidence for the role of physical activity in reducing risk of type 2 diabetes and cardiovascular disease'. En Journal of Applied Physiology 99, 1193-1204.

Dahmann N., Wolch J., Joassart-Marcelli P., Reynolds K., Jerrett M. (2010). 'The active city? Disparities in provision of urban public recreation resources'. En Health \& Place 16, 431-445.

Gómez-Restrepo C., Bohórquez A., Pinto D., Gil Laverde J., Rondon M., Díaz-Granados N. (2004). Prevalencia de depresión y factores asociados con ella en la población colombiana'. Rev Panam Salud Publica, 16(6), 378386.

Hill J., Wyatt H. (2005). 'Role of physical activity in preventing and treating obesity'. En Journal of Applied Physio$\log y 99,765-770$.

Horton R. (2005). The negected epidemic of chronic disease, Lancet, 366 (9496) 1514.

Instituto Nacional de Salud (2005). Encuesta Nacional de la situación nutricional en Colombia ENSIN.

Kien C., Chiodo A. (2003). 'Physical activity in middle school-aged children participating in a school-based recreation program'. En Arch Pediatr Adolesc Med, 157, 811-815.

Kruger J. (2008). 'Parks, Recreation, and Public Health Collaborative'. En Environ Health Insights, 2, 123-125.

León H., Melo C., Zea A. (2009). 'La concepción del sedenta- 
rismo y sus consecuencias'. En Sol de Aquino, 7, 49-56.

Lloyd-Jones D., Adams R., Carnethon M., De Simone G., Ferguson B., Flegal K., et al. (2009). Heart association statistics committee and stroke statistics subcommittee heart disease and stroke statistics 2009, Update: A Report From the American Circulation, 119, 480-486.

Lucumí D., Gutiérrez A., Moreno J., Gómez L., Lagos N., Rosero M., et al. (2008). 'Planeación local para enfrentar el desafío de las enfermedades crónicas en Pasto Colombia'. En Rev. Salud Pública, 10(2), 343-351.

Mathieu P., Poirier P., Pibarot P., Lemieux I., Després J. (2009). Visceral obesity: the link among inflammation, hypertension, and cardiovascular disease, Hypertension, 53, 577-584.

Ministerio de Protección Social (2008). Indicadores básicos 2008, situación de la salud en Colombia.

Murphy N., Carbone P. (2008). 'Promoting the Participation of Children With Disabilities in Sports, Recreation, and Physical Activities'. En Pediatrics 121(5), 1057-1061.

Norman G., Schmid B., Sallis J., Calfas K., Patrick K. (2005). 'Psychosocial and environmental correlates of adolescent sedentary behaviors'. En Pediatrics 116, 908916.

Ott M., Browne G., Byrne C., Roberts J., Gatni A., Baterman A. (2006). 'Recreation for children on social assistan- ce, 4-17 years old, pays for itself the same year'. En J. Public Health, 28(3), 203-208.

Petryshen P., Hawkins J., Fronchak T.. (2001). 'An evaluation of the social recreation component of a community mental health program'. En Psychiatr Rehabil J, 24(3), 293-298.

Richardson C., Faulkner G., McDevitt J., Skrinar G., Hutchinson D., Piette J. (2005). 'Integrating physical activity into mental health services for persons with serious mental illness'. En Psychiatric Services, 56(3), 324-331.

Rogers M., Zaragoza-Lao E. (2003). 'Happiness and children's health: an investigation of art, entertainment, and recreation Am'. En J. Public Health, 93(2), 288-289.

Thompson P., Buchner D., Piña I., Balady G., Williams M., Marcus B., et al. (2003). Exercise and physical activity in the prevention and treatment of atherosclerotic cardiovascular disease, Circulation, 107, 3109-3116.

Todd J., Robinson R. (2003). 'Osteoporosis and exercise'. En Med. J. 79, 320-323.

Warburton D., Nicol C., Bredin S. (2006). 'Health benefits of physical activity: the evidence'. En CMAJ 174(6), 801-809. 